\title{
OBSERVATIONS OF OB STELLAR ASSOCIATIONS WITH THE SPACE TELESCOPE
} GLAZAR

\author{
H.M. TOVMASSIAN ${ }^{1}$, R.Kh. HOVHANNESSIAN ${ }^{2}$, R.A. EPREMIAN ${ }^{2}$ and D. \\ HUGUENIN $^{3}$ \\ ${ }^{1}$ Byurakan Astrophysical Observatory, Armenia, and \\ INAOE, Mexico \\ ${ }^{2}$ Byurakan Astrophysical Observatory, Armenia \\ ${ }^{3}$ Geneva Observatory, Switzerland
}

Observations in the directions of about a dozen OB stellar associations have been carried out with the UV space telescope Glazar, which has been functioning at the Mir Space Station since 1987.

The field of view of the telescope is 1.3 . Observations were made at $1640 \AA$ with a passband of about $250 \AA$. As detector, a Micro Channel Intensifier and a film sensitive to visible light was used. Observations were made without using star trackers, relying only on the stabilization accuracy of the whole Space Station Mir, which was maintained with about 1 arcmin in a given direction at the inertial orientation of the Station. The limiting stellar magnitude of observations at these conditions was about $11^{\mathrm{m}}$ at $1640 \AA$ in the initial period of observations and declined by about 2.5 in about two years.

Observations made with the Glazar allowed the detection in the observed fields of $-15 \%$ new, unknown OB stars, the discovery of hot components of some late type stars, and the detailed study of the distribution of $O B$ stars along line of sight, which resulted in the detection of new OB stellar associations. The reason for the latter is that the larger effect of absorption by interstellar dust on the emission of observed stars at $1640 \AA$ resulted in larger dispersions in determined distances of stars and thus allowed the existence of different groupings of stars to be better revealed.

As a result of observations made with the Glazar, a new type of stellar associations, consisting of stars of spectral types later than B3, have been detected.

The results of observations in the directions of stellar associations in the Ori, Per, Sco, Cass, Gem, Mon and Pup have already been published. In this report we present the results of observations in Vela, Car and Aur.

Vela. Three groups of stars at distances $460 \mathrm{pc}$, (a B association) (Tovmassian 1991), $1100 \mathrm{pc}$ and $1700 \mathrm{pc}$ (OB associations) were detected. To avoid the existing confusion in naming of stellar associations in this region by other investigators we suggest their designation by distances expressed in kpc, i.e. Vela B 0.5, Vela OB 1.1 and Vela OB 1.7.

A new $O$ type star cluster with at least $11-12$ OB stars with a diameter of $\sim 1^{\circ}$ centred at $(1, b)=(246.6,-1.8)$ has been discovered (Tovmassian 1991$)$.

The dust in the association at $1100 \mathrm{pc}$ is concentrated in separate, large clouds. The space between associations is free of dust. 
Carina. Six associations at distances $600,1100,2000,3000,4000$ and $5600 \mathrm{pc}$ were discovered. The one at $600 \mathrm{pc}$ and another probable one at $300 \mathrm{pc}$ are $\mathrm{B}$ associations. The associations detected are designated as Car B 0.3 (?), Car B 0.6, Car OB 1.1, Car OB 2.0, Car OB 3.0, Car OB 4.0 and Car OB 5.6. The Carina nebula together with four clusters, $\operatorname{Tr} 14, \operatorname{Tr} 16, \mathrm{Cr} 228$ and $\mathrm{Cr} 232$ is in the content of the stellar association Car OB 2.0 at a distance of $2200 \mathrm{pc}$. Only two stellar associations, Car OB 1 and Car OB 2 with estimated distances 2500-3000 pc and 2000-3200 pc respectively were previously known in this region.

The distribution of the dust in all associations is patchy and there is no absorbing matter in the space between them.

Auriga. Four stellar associations were detected here. Two definite ones are at distances 1100 pc and $2000 \mathrm{pc}$. The distance of the first one coincides well with the known distance of the stellar association Aur OB 1. Two other suspected associations with only three stars in each of them are at distances $600 \mathrm{pc}$ and $3000 \mathrm{pc}$. The latter value coincides with the known distance of the association Aur $\mathrm{OB} 2$.

Thus, instead of two known stellar associations here the observations with the Glazar revealed the existence of two more groups, one of which is placed between known OB stellar associations, and the other one is a nearby $B$ association.

\section{Acknowledgements}

HMT acknowledges Conacyt (Mexico) for partial support of this work.

\section{References}

Tovmassian, H.M., Khodjayants, Yu.M., Krmoyan, M.N., Kashin, A.L., Zakharian, A.Z., Hovhannessian, R.Kh., Mkrtchian, M.A., Tovmassian, G.H., Huguenin, D., Bootov, V.V., Romanenko, Yu.V., Laveikin, A.I. and Alexandrov, A.P., 1988. Pis'ma v AZh, 14, 291.

Tovmassian, H.M., 1991. Astrofisika, 35, 679. 